\title{
A Stable Protein - CutA1
}

\author{
Azumi Hirata1,2, Aya Sato², Takashi Tadokoro², Yuichi Koga², \\ Shigenori Kanaya ${ }^{2}$ and Kazufumi Takano ${ }^{1,2}$ \\ ${ }^{1}$ Department of Biomolecular Chemistry, \\ Kyoto Prefectural University, \\ ${ }^{2}$ Department of Material and Life Science, \\ Osaka University, \\ Japan
}

\section{Introduction}

CutA1 is a universal protein distributed in bacteria, plants and animals, including humans. This protein was originally isolated and characterized from a gene locus of Escherichia coli called cutA (Fong et al., 1995). The CutA protein is involved in copper tolerance; moreover, it affects divalent cation tolerance levels of zinc, nickel, cobalt and cadmium salts. The cutA locus consists of two operons: one containing a single gene encoding a cytoplasmic protein, CutA1, and the other is composed of two genes encoding the inner-membrane proteins, CutA2 and CutA3. It has been reported that mammalian CutA1 is necessary for anchoring the enzyme acetylcholinesterase (AChE) in neuronal cell membranes (Navaratnam et al., 2000; Perrier et al., 2000). Additionally, CutA1 affects the folding, oligomerization, and secretion of AChE (Falasca et al., 2005; Liang et al., 2009), and co-expression with CutA1 increased the formation and secretion of AChE tetramers (Liang et al., 2009). Therefore, the presence of CutA1 in the secretory pathway affects the processing, probably the folding, and the oligomerization of AChE. However, the precise functions of CutA1 remain to be validated.

The structure of bacterial and mammalian CutA1 has been crystallographically demonstrated in Pyrococcus horikoshii (Ph-CutA1) (Tanaka et al., 2004a), Thermus thermophilus (Tt-CutA1) (Tanaka et al., 2006), Oryza sativa (Os-CutA1) (Sawano et al., 2008), Escherichia coli (Ec-CutA1) (Arnesano et al., 2003), Homo sapiens (Hs-CutA1) (Bagautdinov et al., 2008), Thermotoga maritima (Tm-CutA1) (Savchenko et al., 2004), and Xanthomonas campestris (Xc-CutA1) (Lin et al., 2006). In all cases, from bacterial to mammalian, the trimeric structure of CutA1 was conserved. Moreover, the heatdenaturation temperatures of Ph-CutA1, Tt-CutA1, Os-CutA1, Es-CutA1, and Hs-CutA1 are quite high: $150,113,97,90$, and $96^{\circ} \mathrm{C}$, respectively. The denaturation temperature of Ph-CutA1 $\left(150^{\circ} \mathrm{C}\right)$ is among the highest of known proteins. Several factors responsible for the extreme thermostability of proteins have been proposed, such as an increase in the number of ion pairs and hydrogen bonds (Aguilar et al., 1997; Perutz \& Raidt, 1975; Tahirov et al., 1998; Tanner et al., 1996; Yamagata et al., 2001), core hydrophobicity (Schumann et al., 1993; Takano et al., 1995), packing density (Russell et al., 1994), as well 
as the oligomerization of several subunits (Dams \& Jaenicke, 1999; Jaenicke et al., 1996; Sterner et al., 1996), and entropic effects due to relatively shorter surface loops and peptide chains (Russell et al., 1994; Yamagata et al., 2001). The difference in stability among CutA1 was explained by the difference in electrostatic interactions (Matsuura et al., 2010; Sawano et al., 2008; Tanaka et al, 2004a; Tanaka et al., 2006) and the conformation of the $\beta 2$-strand (Bagautdinov et al., 2008; Savchenko et al., 2004; Tanaka et al, 2004a). Though their stabilities fundamentally depended on the optimal growth temperatures of their host organisms, their denaturation temperatures were remarkably higher than the optimal growth temperatures. These results suggested that the unique trimeric structural motif, which enables tightly intertwined subunit interactions among the $\beta$-strands, was the critical factor in the unusually high stability of CutA1.

Recently, we cloned the gene encoding CutA1 from Shewanella sp. strain SIB1 (SIB1-CutA1), overexpressed it in E. coli, purified the recombinant protein and crystallized it (Sato et al., 2011). Shewanella sp. SIB1 is a psychrophilic bacterium that grows rapidly at $20^{\circ} \mathrm{C}$ (Kato et al., 2001). Although this bacterium can grow at temperatures as low as $-0.15^{\circ} \mathrm{C}$, growth was inhibited at temperatures exceeding $30^{\circ} \mathrm{C}$. We demonstrated that the heat-denaturation temperature of SIB1-CutA1 was $95^{\circ} \mathrm{C}$, suggesting that SIB1-CutA1 needs to maintain high stability in order to function, even in psychrophilic organisms. However, the precise function of Cut-A1 remains controversial.

In this chapter, we discuss the robustness of CutA1 from the viewpoint of its structure. Herein, we first briefly introduce the structure and stability of Ph-CutA1, Tt-CutA1, OsCutA1, Tm-CutA1, Es-CutA1, Hs-CutA1, and SIB1-CutA1. Next, we will further discuss why the stability of CutA1 is so structurally remarkable using our novel results of CutA1 from the psychrophilic bacterium Shewanella oneidensis MR-1 (So-CutA1).

\section{Structure and stability of CutA1 proteins}

\subsection{CutA1 from Pyrococcus horikoshii}

Pyrococcus horikoshii OT3, a hyperthermophilic obligate anaerobe, can grow at temperatures between 88 and $104^{\circ} \mathrm{C}$ with an optimal temperature of $98^{\circ} \mathrm{C}$. Differential scanning calorimetry (DSC) analysis revealed that the denaturation peak of Ph-CutA1 appeared near $150^{\circ} \mathrm{C}$ at $\mathrm{pH} 7.0$, indicating that the folding of Ph-CutA1 was maintained near this temperature and $\mathrm{pH}$ (Tanaka et al., 2006). Interestingly, this temperature was $50^{\circ} \mathrm{C}$ higher than the optimum growth temperature of the organism.

The crystal structure of Ph-CutA1 consisted of a tightly intertwined trimer assembled so as to form a closed circular $\beta$-sheet structure (Fig. 1). The monomeric structure consisted of three $\alpha$-helices and five $\beta$-strands. Two short $(\beta 1$ and $\beta 4)$ and two long ( $\beta 2$ and $\beta 3) \beta$-strands formed an askew curved sheet. The trimer was composed of monomers through interactions between the edges of three $\beta$-strands. Each trimer had three identical intersubunit interfaces, in which both edges of one strand ( $\beta 2)$ interacted with the edges of the $\beta 2$-strand in the other two subunits, and one short strand $(\beta 5)$ mutually interacted with the $\beta 4$-strand of a different subunit. This tightly intertwined interaction appeared to contribute to the stabilization of the trimeric structures of the $\mathrm{Ph}-\mathrm{CutA} 1$ protein. 


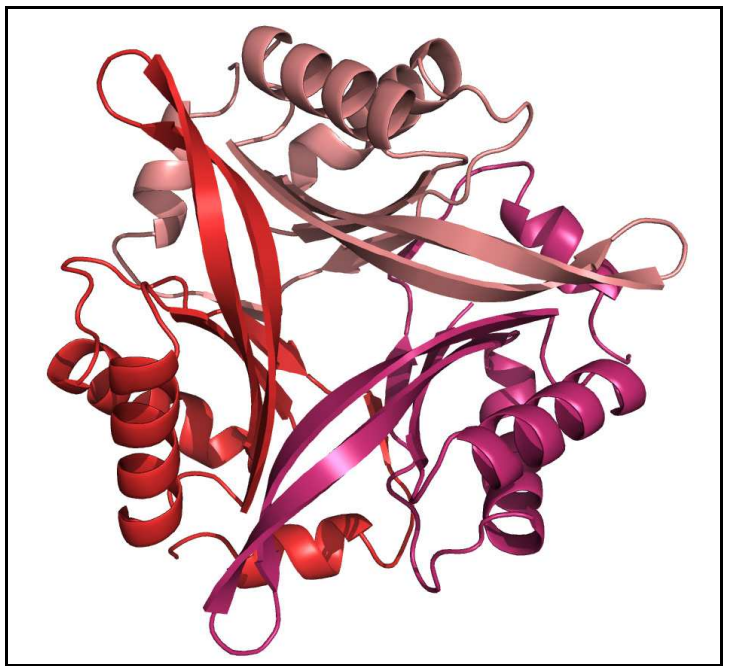

Fig. 1. Ribbon diagram of trimeric structure of Ph-CutA1 (PDB code 1J2V) at $2.00 \AA$ resolution. The figure was prepared using PyMol (DeLano, 2004).

The structural characteristics of Ph-CutA1 revealed that Ph-CutA1 had many ionizable residues, though it had few neutral residues. The number of ionizable residues (Asp, Glu, Lys, Arg, and His) and intra-subunit ion pairs in Ph-CutA1 were prominent (43 and 30, respectively) compared to those in Ec-CutA1 (25 and 1, respectively). Importantly, the monomeric structure of Ph-CutA1 was highly stabilized by hydrogen bonds, ion pairs, and hydrophobic interactions (Tanaka et al., 2006). The intra-subunit ion pairs consisted of 14 donor and 16 acceptor residues, which were partially exposed to the solvent. Six donor positively-charged residues formed ion pairs between different secondary segments in the same subunit (Arg82-Glu59, His35-Glu50, Arg36-Glu47, Arg68-Glu24, Arg25-Glu99, and Lys101-Glu64), where the other residues formed pairs within the same segment. All of the donor residues (Lys19, Lys66, Lys70, and Arg36) forming inter-subunit ion pairs shared intra-subunit ion pairs that formed of inter- and intra-subunit ion pair networks.

Chemical denaturation by guanidine $\mathrm{HCl}(\mathrm{GdnHCl})$ or urea has been widely used to study protein folding and stability. These denaturants can influence not only the protein stability, but also the ensemble of the native structure. Tanaka et al. (2004b) reported the effects of $\mathrm{GdnHCl}$ on Ph-CutA1 through spectroscopic techniques and crystal structure studies. CD spectra results showed no changes in secondary structure of Ph-CutA1 even in 3 to $8 \mathrm{M}$ $\mathrm{GdnHCl}$, indicating that Ph-CutA1 had a rather rigid secondary structure. The crystal structure of Ph-CutA1 in $3 \mathrm{M} \mathrm{GdnHCl}$ was determined at $1.6 \AA$ resolution by the molecular replacement method using native Ph-CutA1 as a search model. The crystal structure of native Ph-CutA1 had a large number of intermolecular hydrogen bonds, of which more than $90 \%$ were retained in $3 \mathrm{M} \mathrm{GdnHCl}$. The disrupted hydrogen bonds were mainly located on the protein surface. Additionally, Ph-CutA1 in the native state showed that the protein had seven ion pairs per monomer. All ion pairs were present in $3 \mathrm{M} \mathrm{GdnHCl}$, even though they were on the protein surface. These observations indicated that the intermolecular interactions of hydrogen bonds and ion pairs of Ph-CutA1 were extraordinary stable. 


\subsection{CutA1 from Thermus thermophilus, Oryza sativa, and Thermotoga maritima}

Thermus thermophilus, Oryza sativa, and Thermotoga maritima is an extreme thermophilic bacterium with an optimum growing temperature of $75^{\circ} \mathrm{C}$. The optimum growth temperature of mesophilic Oryza sativa from the rice plant is $28^{\circ} \mathrm{C}$. Both of the CutA1 proteins from these organisms had a trimeric form (Fig. 2) composed of identical subunits between pH 2.5 and 9.0 (Sawano et al., 2008). DSC analysis has shown that the denaturation temperatures of Tt-CutA1 and Os-CutA1 were $113^{\circ} \mathrm{C}$ at $\mathrm{pH} 7.0$ and $97^{\circ} \mathrm{C}$ at $\mathrm{pH} 7.0$, respectively. These results indicated that both CutA1 proteins had high thermostability. On the other hand, Tt-CutA1 showed higher stability to heat, and moreover, resistance to denaturation than Ph-CutA1 at acidic pH (Tanaka et al., 2006). From a structural standpoint, the number of ionizable residues and intra-subunit ion pairs in Tt-CutA1 is not relatively high (29 and 12, respectively) compared to those in Ph-CutA1 (43 and 30, respectively). These phenomena occurred due to protonated ion pairs, which suggested that the ion pairs in Ph-CutA1 maintained its structural integrity at neutral $\mathrm{pH}$. Changes in the cavity volume in the interior of a protein upon its denaturation affected the conformational stability (Eriksson et al., 1992; Funahashi et al., 2001). The total cavity volumes of the tertiary trimeric structure of Tt-CutA1 were smaller than those of Ph-CutA1. In addition, Ph-CutA1 had a large cavity near the center of a trimer along with four smaller cavities. These observations indicated that Tt-CutA1 formed tighter interactions at the trimer interfaces than Ph-CutA1, resulting in the high stability of Tt-CutA1 at acidic $\mathrm{pH}$.
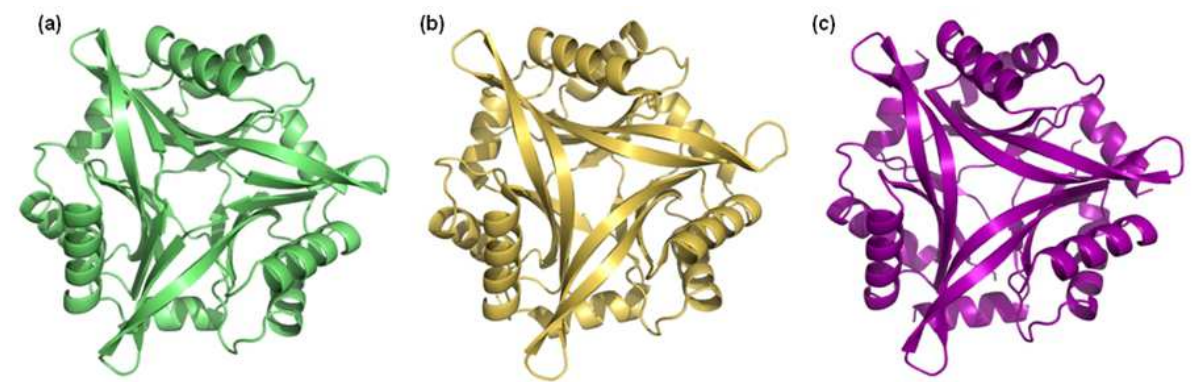

Fig. 2. Ribbon diagrams of trimeric structures of (a) Tt-CutA1 (PDB code 1NZA), (b) OsCutA1 (PDB code 2Z0M) and (c) Tm-CutA1 (PDB code 1O5J) at $1.70 \AA$, $3.02 \AA$, and $1.95 \AA$ resolution, respectively. The figures were generated with PyMol (DeLano, 2004).

The CutA1 from Thermotoga maritima showed amino acid sequence identities to those of EcCutA1 (35\%), Hs-CutA1 (32\%) and Ph-CutA1 (46\%). The structure of Tm-CutA1 was very similar to those of other CutA1 proteins (Fig. 2).

\subsection{CutA1 from Escherichia coli}

The CutA1 protein was originally identified in the cutA gene locus of Escherichia coli, which was involved in divalent metal tolerance. Although the optimal growth temperature of Escherichia coli is $37^{\circ} \mathrm{C}$, the denaturation temperature of Ec-CutA1 is greater than $90^{\circ} \mathrm{C}$ at $\mathrm{pH}$ 9.0, which was also significantly higher than other proteins from mesophilic E. coli.

The structure of Ec-CutA1 consisted of homotrimers (Arnesano et al., 2003) (Fig. 3). In the crystal asymmetric unit, two homotrimers were present that made extensive contacts 
leading to a dimer of trimers. One trimer was rotated by $60^{\circ}$ around the axis perpendicular to the trimer plane with respect to the other.
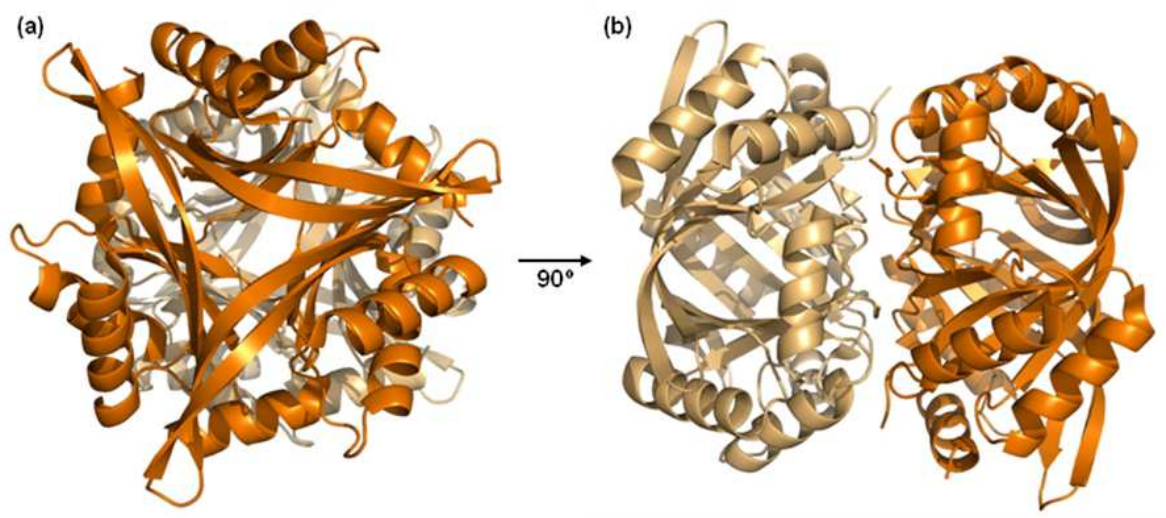

Fig. 3. Ribbon diagram of two trimers of Ec-CutA1 (PDB code 1NAQ) at $1.7 \AA$ A resolution. The structures are shown in two different orientations; (a) front view and (b) side view with $90^{\circ}$ rotation of (a). The figure was prepared with PyMol (DeLano, 2004).

Recently, it was reported that in order to confirm the thermostabilization mechanism, the structure-sequence compatibility between the conformation of Ec-CutA1 and its native sequence was examined using the Stability Profile of Mutant Protein (SPMP) (Matsuura et al., 2010). SPMP analysis is a method to rationally improve the estimation of the conformational stability of a protein (Ota et al., 1995). A pseudo-energy potential derived from a number of Protein Data Bank (http://www.pdb.org/pdb/home/home.do) structures was used in SPMP analysis to predict protein structures and consisted of four elements: packaging of the side-chains, hydration, local structure, and back-bone/side-chain repulsion. From the SPMP scores, seven positions of Ec-CutA1 were mutated to improve protein stability. The mutant Ec-CutA1 proteins were evaluated structurally and for changes in stability by DSC. The crystal structures of these mutant proteins were highly similar to the wild-type Ec-CutA1 structure, while the stabilities of the proteins with mutations at positions 11 and 61 were remarkably improved. The denaturation temperature of single mutant $\mathrm{S} 11 \mathrm{~V}$ and $\mathrm{E} 61 \mathrm{~V}$ were $105^{\circ} \mathrm{C}$ and $103^{\circ} \mathrm{C}$, respectively, and that of the S11V/E61V double mutant was $114^{\circ} \mathrm{C}$. The values of the denaturation temperatures were improved by 15,13 and $24^{\circ} \mathrm{C}$, respectively, suggesting a cumulative effect for each single mutation. The SPMP evaluations were consistent with the DSC results. These observations suggested that these substitutions resulted in changes in the hydration effect, local structure, and side chain packaging. Positions 11 and 61 were located in the $\beta$-sheet and the two substitutions included residues with a higher propensity to form the $\beta$-sheet, which indicated enhanced stabilization (Matsuura et al., 2010).

\subsection{CutA1 from Homo sapiens}

The heat-denaturation temperature of CutA1 from Homo sapiens (Hs-CutA1) was $96^{\circ} \mathrm{C}$, which was remarkably higher than the optimal temperature of Homo sapiens. The N-terminal 
sequence of Hs-CutA1 was hydrophobic and might represent a cleavable secretion signal, a mitochondrial import signal, or a transmembrane anchor (Perrier et al., 2000); whereas, the bacterial CutA1 was devoid of this hydrophobic domain, and Ec-CutA1 was located in the cytoplasm (Arnesano et al., 2003).

Hs-CutA1 was functional as a trimer and the orthorhombic crystal form contained two trimers per asymmetric unit (Bagautdinov et al., 2008). A rigid trimeric core structure appeared to be common to the CutA1 proteins. Similar to other CutA1 structures, the HsCutA1 subunit was made up of a double $\beta a \beta$ motif. Strand $\beta 2$ in Hs-CutA1, however, had a kink at its center that may have been caused by the presence of Pro101 in the middle of the $\beta 2$-strand. This kink-type deformation of $\beta 2$ close to Pro101 allowed the formation of hydrogen bond arrangements that stabilized the $\beta 2$-strand in Hs-CutA1. The conformation of $\beta 2$ may have a possible role in protein thermostability.

The $\beta 2$ - and $\beta 3$-strands and the loop between them (the $\beta 2-\beta 3$ loop) in Hs-CutA1 formed an extended $\beta$-hairpin. The six subunits in the asymmetric unit and the two trimers formed by them were very similar. However, superposition of the Hs-CutA1 subunits showed different conformations in the turn region of the $\beta$-hairpins, indicating a high degree of conformational flexibility. Actually, superposition of CutA1 trimers from different sources indicated that the main differences were observed in the turn area of the $\beta$-hairpins.

The conserved residues were mainly isolated to two regions in the Hs-CutA1 subunit. A loop region between $\alpha 2$ and $\beta 4$, spatially close to the $\beta$-hairpin turn, encompassed the conserved residues His141, Pro142 and Tyr143. The other highly conserved amino acids Cys96 and Tyr107 in $\beta 2$, Glu118, Lys124 and Thr125 in $\beta 3$, Trp109 and Gly111 in the $\beta 2-\beta 3$ loop and Tyr160 and Trp163 in a3 were clustered at the opposite end of the scaffold. The tertiary fold was assembled such that the two conserved regions in the subunits were brought together and formed three potential active sites in the clefts at the trimer interfaces.

In conclusion, the functionally important areas of Hs-CutA1 appeared to be the putative active site clefts and the flexible $\beta$-hairpins. Oligomerization of Hs-CutA1 allowed the small protein to form a compact cylinder-shaped structure and offered the three $\beta$-hairpins and cleft site for specific interactions with other proteins and molecules. These regions contained conserved residues and the flexible $\beta$-hairpin may have changed conformation upon binding of effectors and docking with a receptor. The negatively charged cleft of Hs-CutA1 reflected the positive charge of the substrate ligands and was readily accessible to solvent.

\subsection{CutA1 from Shewanella sp. SIB1}

The CutA1 protein from Shewanella sp. SIB1 (SIB1-CutA1) contained 108 amino acid residues and shared 25, 30, and 39\% identity with Ph-CutA1, Ec-CutA1, and Hs-CutA1, respectively. Recently, we determined the crystal structure of SIB1-CutA1 and measured its thermal stability (Sato et al., 2011).

The overall structure of SIB1-CutA1 was a trimeric structure, which resembled other homologous CutA1 proteins (Fig. 4). The root-mean-square deviations (r.m.s.d.) of the $\mathrm{C}^{a}$ atoms for Ph-CutA1 and Ec-CutA1 against SIB1-CutA1 were 1.08 and $1.00 \AA$ as a monomer, and 1.17 and $1.11 \AA$ as a trimer, respectively. The r.m.s.d. of the $\mathrm{C}^{a}$ atoms between any pair of subunit SIB1-CutA1 was 0.50-0.58 $\AA$. Each subunit consisted of three a-helices and six $\beta$ strands, although the monomers of any other CutA1 had three $\alpha$-helices and five $\beta$-strands. 
The main difference was observed in the $\beta 2$-strand (Figs. 4 and 5). The $\beta 2$-strand was divided into two short $\beta 2$-strands, $\beta 2 \mathrm{a}$ and $\beta 2 \mathrm{~b}$, in SIB1-CutA1. Ph-CutA1 and Tm-CutA1 isolated from hyperthermophiles did not exhibit kinks, whereas Tt-CutA1, Os-CutA1, EcCutA1, and Hs-CutA1 had a conformational kink in the $\beta 2$-strand. This kink was caused from an insertion of a Pro residue into the $\beta 2$-strand. In addition, a Pro deletion variant of Ec-CutA1 increased its stability. A Gln residue (Gln39) was inserted into a $\beta 2$-strand and was divided into two short $\beta$-strands in the SIB1-CutA1 from a psychrotrophic bacterium. So-CutA1 also had an insertion of an Ala residue in the $\beta 2$-strand. Therefore, these results suggested that the conformation of the $\beta 2$-strand affected the thermostability of CutA1 since hyperthermophilic CutA1 had no kink, although mesophilic CutA1 contained a kink, and psychrotrophic CutA1 had two short divided $\beta$-strands. Moreover, hydrogen bonds between strands may have affected the stability. A kink caused by Pro (Pro58) in the $\beta 3$ strand was another peculiarity of SIB1-CutA1. The kink in the $\beta 3$ strand also seemed to be a factor leading the split of the $\beta 2$-strand in SIB-CutA1, since there was interaction between $\beta 2 \mathrm{a}$ and $\beta 2 \mathrm{~b}$ of Gln39 and $\beta 3$ of Pro58.

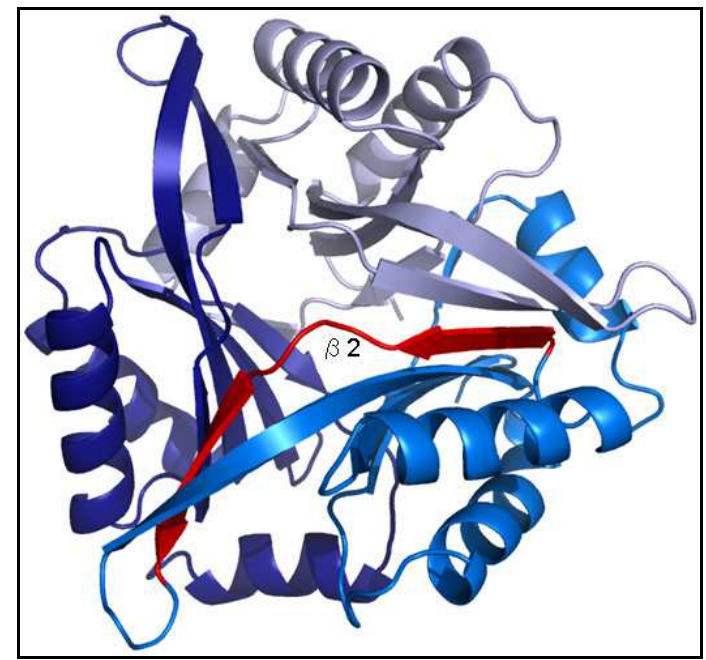

Fig. 4. Ribbon diagram of trimeric structure of SIB1-CutA1 (PDB code 3AHP) at $2.7 \AA$ resolution. The $\beta$-strand is shown in red. The figure was prepared using PyMol (DeLano, 2004).

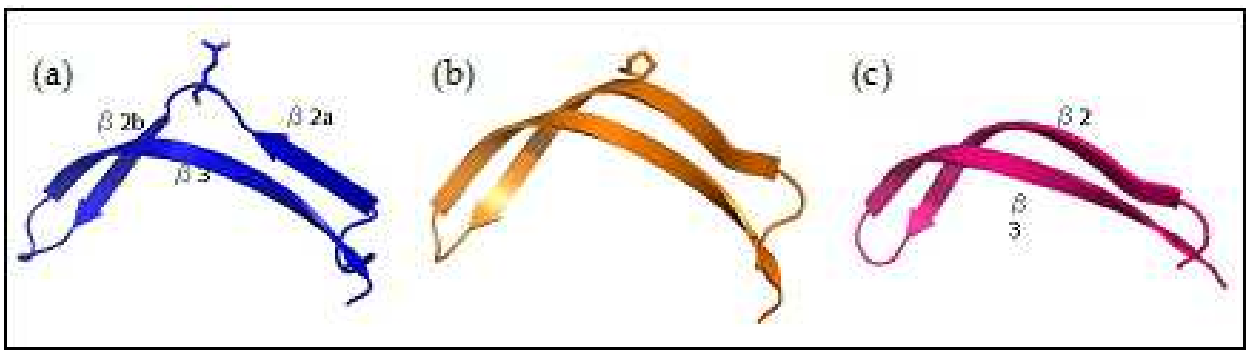

Fig. 5. Close-up views of $\beta 2$ ( $\beta 2$ a and $\beta 2 b$ ) and $\beta 3$. (a) SIB1-CutA1. (b) Ec-CutA1. (c) PhCutA1. The figure was prepared using PyMol (DeLano, 2004). 
The SIB1-CutA1 protein began to unfold at $80^{\circ} \mathrm{C}$ at $\mathrm{pH}$ 7.0. However, the unfolding was not completed at $95^{\circ} \mathrm{C}$, because the $\mathrm{CD}$ value at $95^{\circ} \mathrm{C}$ and $\mathrm{pH} 7.0$ differed from that at $95^{\circ} \mathrm{C}$ and $\mathrm{pH}$ 5.0. Shewanella sp. could not grow at temperatures over $30^{\circ} \mathrm{C}$, and other enzymes from Shewanella sp. SIB1, FKBP22 and ribonuclease H1 were inactivated at temperatures less than $40^{\circ} \mathrm{C}$. These results illustrated the high stability of SIB1-CutA1.

\section{Function of CutA1 proteins from the viewpoint of their structure}

Despite the similarities in folding of copper chaperones and roles in copper tolerance, CutA1 proteins did not possess the classical CXXC motif that has been shown to bind metal ions via thiol sulphurs in Cys residues (Bull \& Cox, 1994; O'Halloran, 1993). In Ec-CutA1, three cysteine residues $(16,39$, and 79$)$ were far apart both in sequence and structure, as none were located within a single subunit or trimer. This suggested that potential metal-binding features of CutA1 were different from those of metallochaperones. Moreover, in Tm-CutA1, there was a cavity accessible from the outside via three solvent accessible channels. The cavity of Tm-CutA1 was formed by a number of conserved aromatic residues (Tyr45, Trp47, Tyr81, and Trp101), and charged residues (Asp54, Glu56, and Glu82), which made this cleft a strong candidate for a conserved function. Several conserved residues including Cys35 covered inner surfaces of the cavity that may serve as potential metal binding sites. Though CutA has been known to be involved in metal homeostasis, the metal-binding CXXC sequence motif was not found in Tm-CutA1.

The CutA1 architecture was similar to those of several well-characterized PII signal transducer proteins (Arnesano et al., 2003; Savchenko et al., 2004). PII proteins are trimeric structures and integrate intracellular nitrogen and carbon status signals to the control of enzymes responsible for nitrogen assimilation (Ninfa \& Atkinson, 2000). The trimeric PIIlike domains were reported to act as signal sensors that regulated unknown catalytic activities of more conserved domains (Godsey et al., 2007; Saikatendu et al., 2006), which might suggest that the conserved trimeric assembly showed a similar mechanism of action as PII proteins and CutA1. On the other hand, the major structural differences included the presence of a C-terminal $\beta$-strand in PII proteins, while in CutA1 they replaced the a3helices. Additionally, a large loop between strands $\beta 2$ and $\beta 3$ protruded into the solvent, whereas strands $\beta 2$ and $\beta 3$ in CutA1 were longer and formed $\beta$-hairpin strands. In PII proteins, the two central $\beta$-strands ( $\beta 3$ and $\beta 4$ ) were joined by the T-loop, which were composed of 17 amino acids and played a key role in protein-protein interactions. In TmCutA1, the $\beta 3$ - and $\beta 4$-strands were joined by a two amino acid turn to form a hairpin loop. The residues in the C-terminal extension of the Tm-CutA1 $\beta 3$-strand (Tyr45, Trp46, and Trp47) were conserved. However, the structure of this region in Tm-CutA1 was different from that in PII proteins, suggesting that this region in Tm-CutA1 may not participate in protein-protein interactions (Savchenko et al., 2004).

Recently, it was shown that overexpressed CutA isoform2 sensitized HeLa cells to copper toxicity by promoting copper-induced apoptosis (Yang et al., 2008); moreover, the inhibitory effect of excessive copper on cell proliferation was enhanced by over-expression of CutA isoform2. Based on these results, it was suggested that CutA isoform2 was implicated in an important role in copper toxicity. These studies will be helpful to guide future investigations in understanding the physiological role of the CutA1 protein. 


\section{High stability of the trimeric structural motif of CutA1 proteins}

CutA1 has an extraordinary stability that fundamentally depends on the optimal growth temperatures of their host organisms. The difference in stability among CutA1 proteins was explained by the difference in electrostatic interactions and the conformation of the $\beta 2$ strand. From a structural point of view, the high stability of CutA1 originated from the common trimer structural motif. The trimeric structure enabled tightly intertwined interactions among the $\beta$-strands. It was indicated that hydrophobic interactions in $\mathrm{Ph}$ CutA1 were responsible for stabilizing the trimeric structure. Moreover, it was observed that in SIB1-CutA1, the trimer subunits were solidly locked to each other with highly conserved aromatic residues, such as Tyr45, Trp47, Tyr81, Tyr98 and Trp101 (Fig. 6). These residues were located in the subunit interfaces. Therefore, we suggest that increasing stability of CutA1 was caused by the aromatic cluster. There was no report which provided evidence indicating that the aromatic cluster affected the stability of the CutA1 protein.

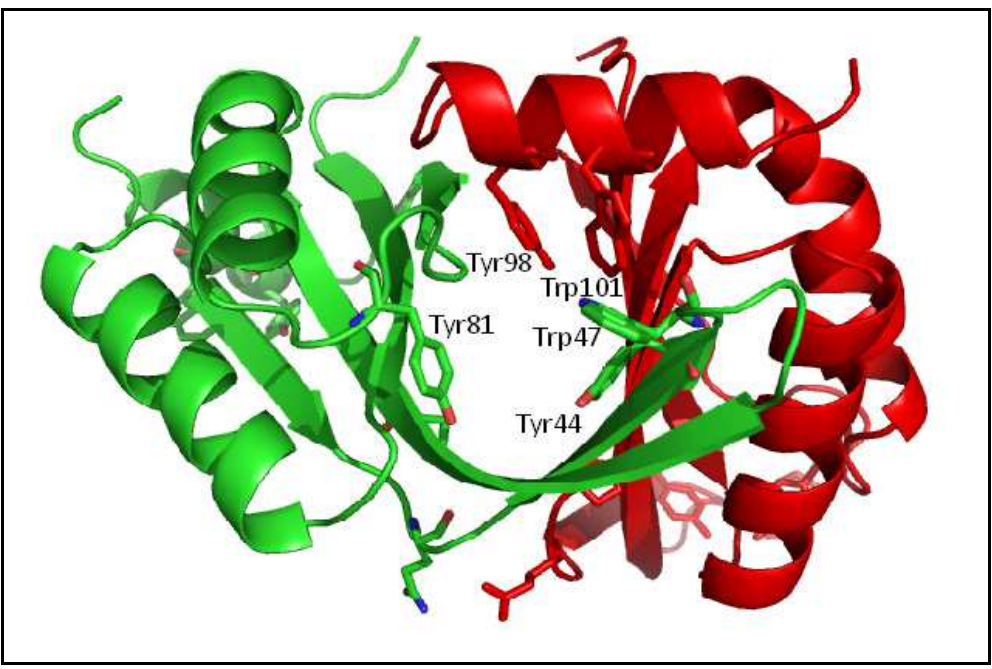

Fig. 6. Close-up view of the subunit interface of SIB1-CutA1. Each monomer is coloured differently. The highly conserved aromatic residues are shown. The figure was prepared using PyMol (DeLano, 2004).

SIB1-CutA1 subunit $A$ ( $B$ or $C$ ) interacted with hydrogen bonds between $\beta 2 \mathrm{a}$ and $\beta 2 \mathrm{~b}$ of subunit $C(A$ or $B)$, between $\beta 2 \mathrm{~b}$ and $\beta 2 \mathrm{a}$ of subunit $B(C$ or $A)$ and between $\beta 4$ and $\beta 5$ of subunit $B(C$ or $A)$, resulting in a tightly intertwined trimer. The intertwined interactions among $\beta$ strands of SIB1-CutA1 appeared to stabilize the trimeric structure as in other CutA1 proteins. Furthermore, three $\alpha$-helices were located on the outside of the trimer and cover $\beta$-strands. Highly conserved aromatic residues, Tyr45, Trp47, and Tyr81 from subunit $A(B$ or $C)$ and Tyr98 and Trp101 from subunit $B(C$ or $A)$ existed in the subunit interfaces. These residues formed a hydrophobic core at the trimer interfaces.

In this section, we discussed our novel results in order to demonstrate the effects of the aromatic cluster on So-CutA1 protein stabilization. The highly conserved aromatic residues 
of So-CutA1 were replaced (Y44A, W46A, Y97A, W100A, and W100F) and the CD spectra measurements and DSC analysis of these mutant proteins were evaluated.

The gene encoding So-CutA1 was amplified by polymerase chain reaction (PCR), where the genomic DNA of Shewanella oneidensis MR-1 was used as a template. The resultant DNA fragment was digested with NdeI and BamHI. Plasmids for overproduction of So-CutA1 were constructed by ligating the resultant DNA fragment into the NdeI-BamHI sites of pET25b. The pET25b derivatives for overproduction of the five mutant proteins were constructed by PCR using the QuikChange II site-directed mutagenesis kit (Stratagene). The pET25b derivative for overproduction of So-CutA1 was used as a template. The mutagenic primers were designed such that the codons for Tyr44, Trp46, Tyr97 and Trp100 were changed to Ala, and the codon for Trp100 was changed to Phe. The resultant DNA fragments were digested with NdeI and $B a m \mathrm{HI}$ and ligated into the NdeI-BamHI sites of pET25b. For overproduction of WT and mutant So-CutA1, E. coli BL21 (DE3) was transformed with WT or mutant pETCutA1 and grown at $37^{\circ} \mathrm{C}$. When $D_{600}$ reached $0.6,1 \mathrm{mM}$ IPTG was added to the culture medium and cultivation was continued at $37^{\circ} \mathrm{C}$ for $4 \mathrm{~h}$. The cells were harvested by centrifugation, disrupted by sonication, and heat-treated at $60^{\circ} \mathrm{C}$ for $10 \mathrm{~min}$. The supernatant was dialyzed against $50 \mathrm{mM}$ Tris- $\mathrm{HCl}$ at $\mathrm{pH} 8.0$ and applied to a HiPrep DEAE column (GE Healthcare Life Sciences). The protein was eluted from the column with a linear gradient of 0 to $1.0 \mathrm{M} \mathrm{NaCl}$ and the purity was analyzed by SDS-PAGE.

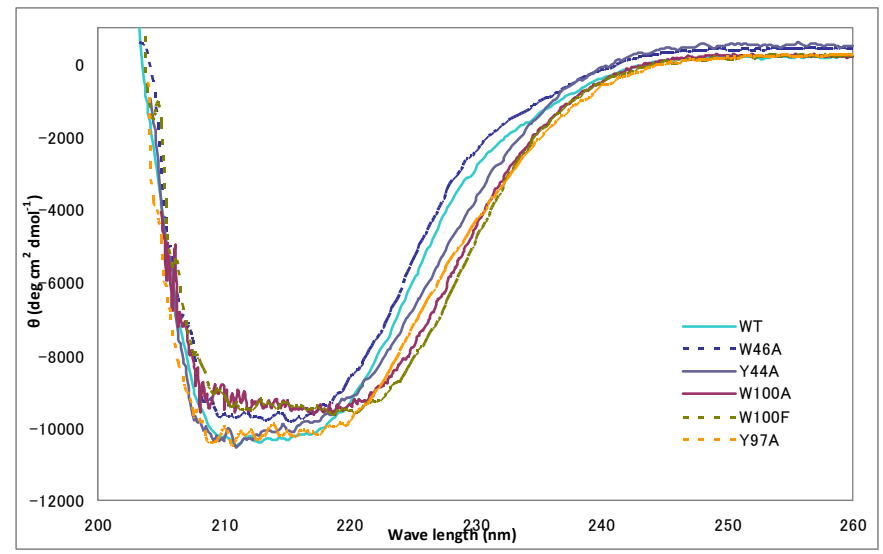

Fig. 7. CD spectra of the WT and mutant So-CutA1 at $\mathrm{pH} 8.0$ and $30^{\circ} \mathrm{C}$.

The CD spectra measurements were made on a J-725 automatic spectropolarimeter from Japan Spectroscopic Co., Ltd. (Tokyo, Japan). The mean residue ellipticity, $\theta$, which has units of degrees $\mathrm{cm}^{2} \mathrm{dmol}^{-1}$, was calculated. For CD spectra measurements, the buffer used consisted of $50 \mathrm{mM}$ Tris- $\mathrm{HCl}$ at $\mathrm{pH} 8.0,1 \mathrm{mM}$ EDTA, and $1 \mathrm{mM}$ DTT. The protein concentration was $0.2 \mathrm{mg} \mathrm{ml}^{-1}$. DSC measurements were carried out on a high-sensitivity VP-DSC controlled by the VPVIEWERTM software package (Microcal, Inc., Northampton, MA, USA) at a scan rate of $1^{\circ} \mathrm{C} \mathrm{min}-1$. The buffer for DSC measurements contained $50 \mathrm{mM}$ Glycine- $\mathrm{NaOH}$ at $\mathrm{pH}$ 9.0, and $1 \mathrm{mM}$ EDTA. The protein concentration during the measurements was $\approx 0.5 \mathrm{mg} \mathrm{ml}^{-1}$. The reversibility of thermal denaturation was verified by reheating the samples. 
The far-UV spectra of these five proteins at $\mathrm{pH} 8.0$ and $30^{\circ} \mathrm{C}$ were nearly similar to those of WT-So-CutA1 as shown in Fig. 7, suggesting they have folded structures. Since far-UV CD spectra of a protein had been affected due to the aromatic residues (Woody, 1994), the differences in far-UV spectra of the six proteins might be caused mainly by differences in the content of aromatic residues. Fig. 8 shows the DSC curves of WT and five mutant proteins at $\mathrm{pH}$ 9.0. The peak temperatures of WT-So-CutA1 was $100^{\circ} \mathrm{C}$, and those of five mutant proteins were 89 (Y44A), 99 (W46A), 85 (Y97A), 90 (W100A), and $94^{\circ} \mathrm{C}(\mathrm{W} 100 \mathrm{~F})$, respectively. The peak temperatures of mutant proteins were lower than that of WT-So-CutA1, suggesting that the aromatic cluster, especially Tyr44, Tyr97, and Trp100, which were located within the interface of the trimer, contributed to the stabilization of So-CutA1 (Fig. 9).

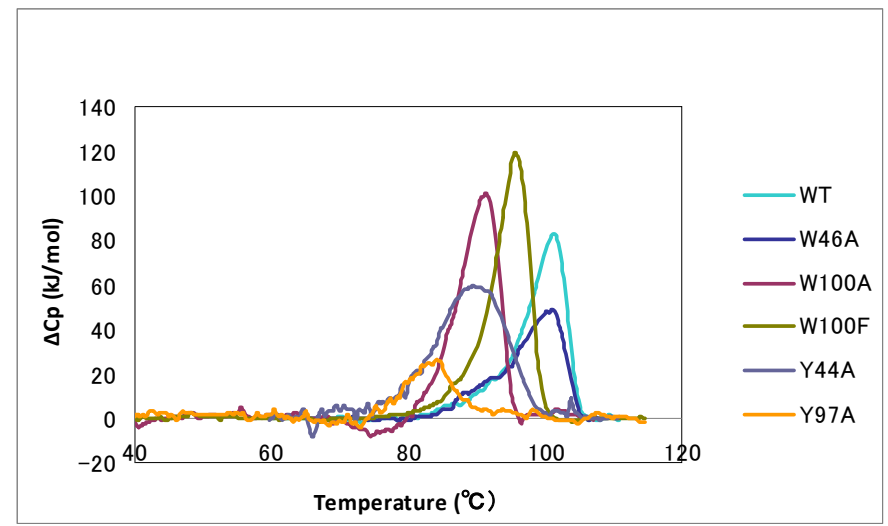

Fig. 8 DSC curves of the WT and mutant So-CutA1 at pH 9.0.

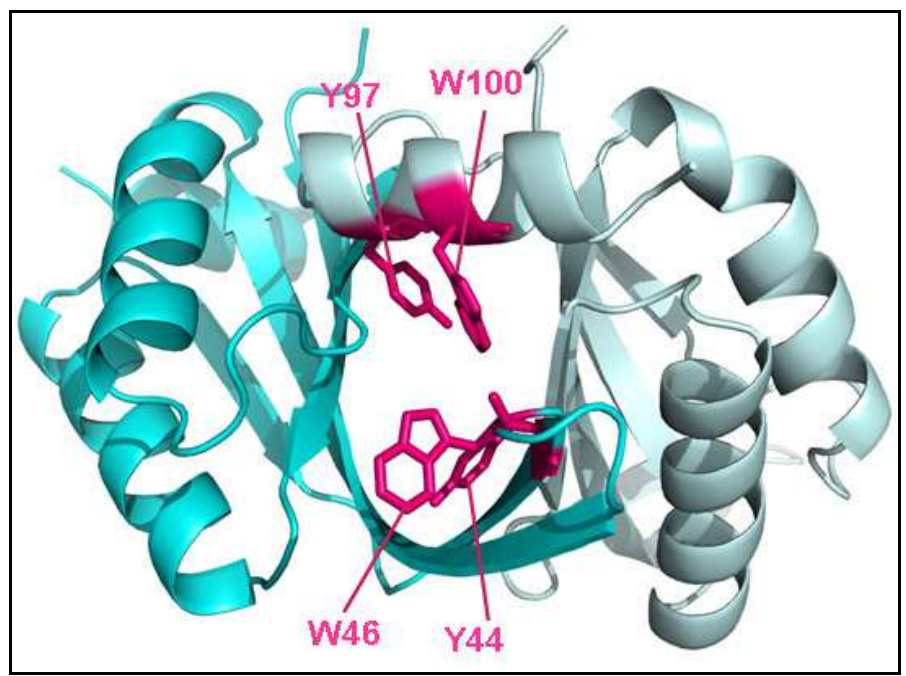

Fig. 9. Close-up view of the subunit interface (model) of So-CutA1. Each monomer is coloured differently. The highly conserved aromatic residues are shown in magenta. The figure was prepared using PyMol (DeLano, 2004). 


\section{Conclusion}

In general, high thermostability of proteins correlated with high resistance against proteolysis. CutA1 unfolded at a remarkably higher temperature compared to the growth temperatures of the host organisms. The stability of CutA1 depended upon the growth temperature. Therefore, CutA1 must maintain the robustness for its function. At this present stage, we cannot explain the relationship between the exact functions and stability of CutA1. Nevertheless, the unusual high stability of CutA1 basically originated from a common trimer structure of these proteins. Structurally, the aromatic cluster, which combined the subunits, participated in the stabilization of CutA1. The evolutionarily conserved trimeric structure and thermostability might point to a similar mechanism of CutA1 action.

\section{Acknowledgment}

This work was supported in part by an Industrial Technology Research Grant Program from the New Energy and Industrial Technology Development Organization (NEDO) of Japan.

\section{References}

Aguilar, C. F.; Sanderson, I.; Moracci, M.; Ciaramella, M.; Nucci, R.; Rossi, M. \& Pearl, L. H. (1997). Crystal Structure of the $\beta$-Glycosidase from the Hyperthermophilic archaeon Sulfolobus solfataricus: Resilience a Key Factor in Thermostability. Journal of Molecular Biology, Vol.271, pp. 789-802, ISSN 0022-2836

Arnesano, F.; Banci, L.; Benvenuti, M.; Bertini, I.; Calderone, V.; Mangani, S. \& Viezzoli, M. S. (2003). The Evolutionarily Conserved Trimeric Structure of CutA1 Proteins Suggests a Role in Signal Transduction. The Journal of Biological Chemistry, Vol.278, No.46, pp. 45999-46006, ISSN 1083-351X

Bagautdinov, B.; Matsuura, Y.; Bagautdinova S.; Kunishima, N. \& Yutani, K. (2008). Structure of Putative CutA1 from Homo sapiens Determined at $2.05 \AA$ Resolution. Acta Crystallographica, Vol.F64, pp. 351-357, ISSN 1744-3091

Bull, P. C. \& Cox, D. W. (1994). Wilson Disease and Menkes Disease: New Handles on Heavy-Metal Transport. Trends in Genetics, Vol.10, pp. 246-252, ISSN 0168-9525

Dams, T. \& Jaenicke, R. (1999). Stability and Folding of Dihydrofolate Reductase from the Hyperthermophilic Bacterium Thermotoga maritima. Biochemistry, Vol.38, pp. 91699178, ISSN 1520-4995

DeLano, W. L. (2004). The PyMOL Molecular Graphics System. DeLano Scientific, San Carlos, California, USA.

Eriksson, A. E.; Baase, W. A.; Zhang, X. J.; Heinz, D. W.; Blaber, M.; Baldwin, E. P. \& Matthews, B. W. (1992). Response of a Protein Structure to Cavity-Creating Mutations and Its Relation to the Hydrophobic Effect. Science, Vol.255, pp. 178-183, ISSN 1095-9203

Falasca, C.; Perrier, N.; Massoulié, J. \& Bon, S. (2005). Determinants of the t Peptide Involved in Folding, Degradation, and Secretion of Acetylcholinesterase. The Journal of Biological Chemistry, Vol.280, No.2, pp. 878-886, ISSN 1083-351X

Fong, S. T.; Camakaris, J. \& Lee, B. T. O. (1995). Molecular Genetics of a Chromosomal Locus Involved in Copper Tolerance in Escherichia coli K-12. Molecular Microbiology, Vol.15, No.6, pp. 1127-1137, ISSN 1365-2958

Funahashi, J.; Takano, K. \& Yutani, K. (2001). Are the Parameters of Various Stabilization Factors Estimated from Mutant Human Lysozymes Compatible with Other Proteins? Protein Engineering, Vol.14, No.2, pp. 127-134, ISSN 1741-0134 
Jaenicke, R.; Schurig, H.; Beaucamp, N. \& Ostendorp, R. (1996). Structure and Stability of Hyperstable Proteins: Glycolytic enzymes from Hyperthermophilic Bacterium. Thermotoga maritima. Advances in Protein Chemistry, Vol.48, pp. 181-269

Kato, T.; Haruki, M.; Imanaka, T.; Morikawa, M. \& Kanaya, S. (2001). Isolation and Characterization of Psychrotrophic Bacteria from Oil-Reservoir Water and Oil sands. Applied Microbiology and Biotechnology, Vol.55, No.6, pp. 794-800, ISSN 1432-0614

Liang, D.; Nunes-Tavares, N.; Carvalho, S. ; Bon, S. \& Massoulié, J. (2009). Protein CutA Undergoes an Unusual Transfer into the Secretory Pathway and Affects the Folding, Oligomerization, and Secretion of Acetylcholinesterase. The Journal of Biological Chemistry, Vol.284, No.8, pp. 5195-5207, ISSN 1083-351X

Lin, C. H.; Chin, K. H.; Gao, F. P.; Lyu, P. C.; Shr, H. L.; Wang, A. H. j. \& Chou, S. H. (2006). Cloning, Crystallization and Preliminary X-Ray Studies of XC2981 from Xanthomonas campestris, a Putative CutA1 Protein Involved in Copper-Ion Homeostasis. Acta Crystallographica, Vol.F62, pp. 1113-1115, ISSN 1744-3091

Matsuura, Y.; Ota, M.; Tanaka, T.; Takehira, M.; Ogasahara, K.; Bagautdinov, B.; Kunishima, N. \& Yutani K. (2010). Remarkable Improvement in the Heat Stability of CutA1 from Escherichia coli by Rational Protein Design. Journal of Biochemistry, Vol.148, No.4, pp. 449-458, ISSN 1756-2651

Navaratnam, D. S.; Fernando, F. S.; Priddle, J. D.; Giles, K.; Clegg, S. M.; Pappin, D. J.; Craig, I. \& Smith, A. d. (2000). Hydrophobic Protein that Copurifies with Human Brain Acetylcholinesterase: Amino Acid Sequence, Genomic Organization, and Chromosomal Localization. Journal of Neurochemistry, Vol.74, pp. 2146-2153, ISSN 1471-4159

Ninfa, A. J. \& Atkinson, M. R. (2000). PII Signal Transduction Proteins. Trends in Microbiology, Vol.8, pp. 172-179, ISSN 0966-842X

O'Halloran, T. V. (1993). Transition Metals in Control of Gene Expression. Science, Vol.261, pp. 715-725, ISSN 1095-9203

Ota, M.; Kanaya, S. \& Nishikawa, K. (1995). Desk-top Analysis of the Structural Stability of Various Point Mutations Introduced into Ribonuclease H. Journal of Molecular Biology, Vol.248, pp. 733-738, ISSN 0022-2836

Perrier, A. L.; Cousin, X.; Boschetti, N.; Haas, R.; Chatel, J. M.; Bon, S.; Roberts, W. L.; Pickett, S. R.; Massoulié, J.; Rosenberry, T. L. \& Krejci, E. (2000). Two Distinct Proteins Are Associated with Tetrameric Acetylcholinesterase on the Cell Surface. The Journal of Biological Chemistry, Vol.275, No.3, pp. 34260-34265, ISSN 1083-351X

Perutz, M. F. \& Raidt, H. (1975). Stereochemical Basis of Heat Stability in Bacterial Ferredoxins and in Haemoglobin A2. Nature, Vol.255, pp. 256-259, ISSN 0028-0836

Russell, R. J. M.; Hough, D. W.; Dansos, M. J. \& Taylor, G. L. (1994). The Crystal Structure of Citrate Synthase from the thermophilic archaeon Thermoplasma acidophilum. Structure, Vol.2, pp. 1157-1167, ISSN 1536-4283

Sato, A.; Yokotani, S.; Tadokoro, T.; Tanaka, S.; Angkawidjaja, C.; Koga, Y.; Takano, K. \& Kanaya, S. (2011). Crystal Structure of Stable Protein CutA1 from Psychrotrophic Bacterium Shewanella sp. SIB1. Journal of Synchrotron Radiation, Vol.18, pp. 6-10, ISSN 0909-0495

Savchenko, A.; Skarina, T.; Evdokimova, E.; Watson, J. D.; Laskowski, R.; Arrowsmith, C. H.; Edwards, A. M.; Joachimiak, A. \& Zhang, R. G. (2004). X-Ray Crystal Structure of CutA from Thermotoga maritima at $1.4 \AA$ Resolution. Proteins: Structure, Function, and Bioinformatics, Vol.54, pp. 162-165, ISSN 1097-0134 
Sawano, M.; Yamamoto, H.; Ogasahara, K.; Kidokoro, S.; Katoh, S.; Ohmura, T.; Katoh, E.; Yokoyama, S. \& Yutani, K. (2008). Thermodynamic Basis for The Stabilities of Three CutA1s from Pyrococcus horikoshii, Thermus thermophilus, Oryza sativa, with Unusually High Denaturation Temperatures. Biochemistry, Vol.47, pp. 721-730, ISSN 1520-4995

Schumann, J.; Bohm, G.; Schumacher, G.; Rudolph, R. \& Jaenicke, R. (1993). Stabilization of Creatinase from Pseudomonas putida by Random mutagenesis. Protein Science, Vol.10, pp. 1612-1620, ISSN 0961-8368

Sterner, R.; Kleemann, G. R.; Szadkowski, H., Lustig, A.; Hennig, M. \& Kirchner, K. (1996). Phosphoribosyl Anthranilate Isomerase from Thermotoga maritima is Extremely Stable and Active Homodimer. Protein Science, Vol.5, pp. 2000-2008, ISSN 0961-8368

Tahirov, T. H.; Oki, H.; Tsukihara, T.; Ogasahara, K.; Yutani, K.; Ogata, K.; Izu, Y.; Tsunasawa, S. \&Kato, I. (1998). Crystal Structure of Methionine Aminopeptidase from Hyperthermophile, Pyrococcus furiosus. Journal of Molecular Biology, Vol.284, pp. 101-124, ISSN 0022-2836

Takano, K.; Ogasahara, K.; Kaneda, H.; Yamagata, Y.; Fujii, S.; Kanaya, E.; Kikuchi, M.; Oobatake, M. \& Yutani, K. (1995). Contribution of Hydrophobic Residues to the Stability of Human Lysozyme: Calorimetric Studies and X-ray Structural Analysis of the Five Isoleucine to Valine Mutants. Journal of Molecular Biology, Vol.254, pp. 62-76, ISSN 0022-2836

Tanaka, T.; Sawano, M.; Ogasahara, K.; Sakaguchi, Y.; Bagautdinov, B.; Katoh, E.; Kuroishi, C.; Shinkai, A.; Yokoyama, S. \&Yutani, K. (2006). Hyper-Thermostability of CutA1 Protein, with a Denaturation Temperature of Nearly $150{ }^{\circ}$ C. FEBS Letters Vol.580, pp. 4224-4230, ISSN 0014-5793

Tanaka, Y.; Tsumoto, K.; Nakanishi, T.; Yasutake, Y.; Sakai, N., Yao, M., Tanaka, I. \& Kumagai, I. (2004a). Structural Implications for Heavy Metal-Induced Reversible Assembly and Aggregation of a Protein: The Case of Pyrococcus horikoshii CutA ${ }^{1}$. FEBS Letters Vol.556, pp. 167-174, ISSN 0014-5793

Tanaka, Y.; Tsumoto, K.; Umetsu, M.; Nakanishi, T.; Yasutake, Y.; Sakai, N., Yao, M., Tanaka, I.; Arakawa, T. \& Kumagai, I. (2004b). Structural Evidence for GuanidineProtein Side Chain Interactions: Crystal Structure of CutA from Pyrococcus horikoshii in 3M Guanidine Hydrochloride. Biochemical and Biophysical Research Communications, Vol.323, pp. 185-191, ISSN 0006-291X

Tanner, J.; Hecht, R. M. \& Krause, K. L. (1996). Determinants of Enzyme Thermostability Observed in the Molecular Structure of Thermus aquaticus D-Glyceraldehyde-3Phosphate Dehydrogenase at $2.5 \AA$ Resolutuin. Biochemistry, Vol.35, pp. 2597-2609, ISSN 1520-4995

Yamagata, Y.; Ogasahara, K.; Hioki, Y.; Lee, S. J.; Nakagawa, A.; Nakamura, H.; Ishida, M.; Kuramitsu, S. \& Yutani, K. (2001). Entropic Stabilization of the Tryptophan Synthase a-Subunit from a Hyperthermophile, Pyrococcus furiosus. X-Ray Analysis and Calorimetry. The Journal of Biological Chemistry, Vol.276, No.14, pp. 11062-11071, ISSN 1083-351X

Yang, J.; Li, Q.; Yang, H.; Yan, L. ; Yang, L. \& Yu, L. (2008). Overexpression of Human CutA1 Isoform2 Enhances the Cytotoxicity of Copper to Hela Cells. Acta Biochemica Polonica, Vol.55, No.2, pp. 411-415, ISSN 1734-154X

Woody, R. W. (1994). Contributions of Tryptophan Side Chains to the Far-Ultraviolet Circular Dichroism of Protein. European Biophysics Journal, Vol.23, pp. 253-262, ISSN 1432-1017 


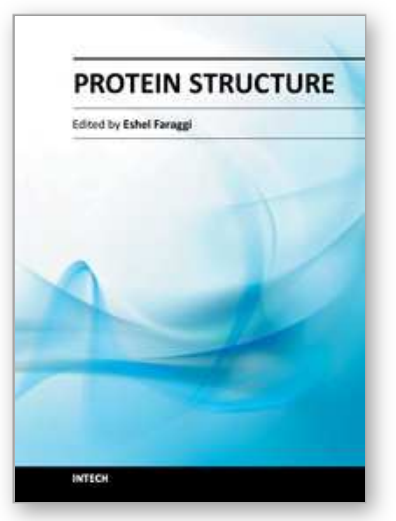

\author{
Protein Structure \\ Edited by Dr. Eshel Faraggi
}

ISBN 978-953-51-0555-8

Hard cover, 396 pages

Publisher InTech

Published online 20, April, 2012

Published in print edition April, 2012

Since the dawn of recorded history, and probably even before, men and women have been grasping at the mechanisms by which they themselves exist. Only relatively recently, did this grasp yield anything of substance, and only within the last several decades did the proteins play a pivotal role in this existence. In this expose on the topic of protein structure some of the current issues in this scientific field are discussed. The aim is that a non-expert can gain some appreciation for the intricacies involved, and in the current state of affairs. The expert meanwhile, we hope, can gain a deeper understanding of the topic.

\title{
How to reference
}

In order to correctly reference this scholarly work, feel free to copy and paste the following:

Azumi Hirata, Aya Sato, Takashi Tadokoro, Yuichi Koga, Shigenori Kanaya and Kazufumi Takano (2012). A Stable Protein - CutA1, Protein Structure, Dr. Eshel Faraggi (Ed.), ISBN: 978-953-51-0555-8, InTech, Available from: http://www.intechopen.com/books/protein-structure/a-stable-protein-cuta1

\section{INTECH}

open science | open minds

\section{InTech Europe}

University Campus STeP Ri

Slavka Krautzeka 83/A

51000 Rijeka, Croatia

Phone: +385 (51) 770447

Fax: +385 (51) 686166

www.intechopen.com

\section{InTech China}

Unit 405, Office Block, Hotel Equatorial Shanghai

No.65, Yan An Road (West), Shanghai, 200040, China 中国上海市延安西路65号上海国际贵都大饭店办公楼405单元

Phone: +86-21-62489820

Fax: $+86-21-62489821$ 
(C) 2012 The Author(s). Licensee IntechOpen. This is an open access article distributed under the terms of the Creative Commons Attribution 3.0 License, which permits unrestricted use, distribution, and reproduction in any medium, provided the original work is properly cited. 